\title{
Entropia na determinação da concentração de sedimentos em suspensão em reservatório
}

\author{
Entropy for determining the concentration \\ of sediment in suspension in reservoir
}

Patrícia Diniz Martins', Bruno Bernardo dos Santos², Frederico Fábio Mauad³, Cristiano Poleto 4

$\square$

\section{RESUMO}

O sedimento encontrado na seção transversal de rios ou reservatórios é resultado das interações ocorridas em uma bacia hidrográfica, sejam elas decorrentes de fatores naturais ou antrópicos. Apesar da quantidade de sedimentos no rio ser o resultado dos processos que acontecem dentro da bacia, os métodos de estimativa de sedimentos baseados nessas características, ou seja, métodos determinísticos, apresentam grandes variações devido ao grande número de variáveis interferentes. Aqui foi aplicada a teoria da entropia, método não determinístico, para estimar o perfil da concentração de sedimentos a montante, a jusante e no interior de um reservatório, propondo a relação entre dois parâmetros com a finalidade de facilitar a estimativa. Foi verificado que é possível utilizar a teoria da entropia para simular o perfil de concentração de sedimentos em reservatórios. A utilização da relação entre os parâmetros facilita os cálculos, reduz o número de parâmetros modelados e, consequentemente, o tempo computacional, além de representar melhor as variações da concentração de sedimentos ao longo do perfil.

Palavras-chave: modelagem, recursos hídricos, sedimentometria.

\begin{abstract}
The sediment found in the cross section of a river or a reservoir is the result of interactions that occur in a watershed, whether from natural or anthropogenic factors. There are still changes in the riverbed conditions depending on the type of sediment, flow velocity, changes in slope and human interventions such as hydraulic works. Although the amount of sediment in the river is the result of processes that occur within the basin, the estimation methods of sediment based on these characteristics, that is, deterministic methods, vary widely due to the large number of interfering variables. This paper deals with the application of the entropy theory, a non-deterministic method to estimate the profile of sediment concentration upstream, downstream and within a reservoir. A relationship between two parameters in order to facilitate the estimation was proposed. It can be observed that it is possible to apply entropy theory to simulate the sediment concentration profile in reservoirs. The use of the relationship between parameters facilitates calculations, reduces the number of modeled parameters and consequently the computational effort and better represents the variation of sediment concentration along the profile. Keywords: modeling; sedimentometry; water resources.
\end{abstract}

\section{INTRODUÇÃO}

A preocupação com os recursos hídricos tem levado à crescente melhoria nas condições das bacias hidrográficas, visando a reduzir o impacto das atividades humanas na qualidade e na quantidade de água. Essa melhoria só pode ser feita mediante o monitoramento sistemático dos corpos hídricos por meio de um gerenciamento integrado dos recursos hídricos.

Em reservatórios, esse monitoramento é ainda mais importante, pois há necessidade de se medir a concentração de sedimentos e avaliar o assoreamento. Quando são construídas barragens, estas impedem a passagem de boa parte de sedimentos que fica retida, reduzindo a vida útil dos reservatórios. Estimativas erradas do assoreamento podem trazer grandes prejuízos para a geração de energia, para a vida útil do reservatório, para a capacidade de armazenamento e para a regularização de cheias. A complexidade envolvendo os processos sedimentológicos e os elevados custos das campanhas de amostragem de sedimentos têm feito gestores adotarem quantidade de postos e frequência de medições inferiores às desejáveis, o que impossibilita um monitoramento contínuo.

$\square$

'Professora adjunta da Universidade Federal do Triângulo Mineiro (UFTM). Doutora pelo Núcleo de Hidrometria do da Escola de Engenharia da de São Carlos da, Universidade de São Paulo (USP) - São Carlos (SP), Brasil.

2Doutorando da Escola de Engenharia de São Carlos da Universidade de São Paulo (USP) - São Carlos (SP), Brasil.

${ }^{3}$ Coordenador do Programa de Pós-Graduação em Ciências da Engenharia Ambiental da Escola de Engenharia de São Carlos da Universidade de São Paulo (USP) - São Carlos (SP), Brasil. ${ }^{4}$ Pesquisador do Programa de Pós-Graduação em Recursos Hídricos e Saneamento Ambiental do Instituto de Pesquisas Hidráulicas da Universidade Federal do Rio Grande do Sul (UFRGS) e do Programa de Pós-Graduação em Ciências da Engenharia Ambiental da Universidade de São Paulo (USP) - São Carlos (SP), Brasil. Endereço para correspondência: Patrícia Diniz Martins - Avenida Dr. Randolfo Borges Junior, 1.250 - Univerdecidade - 38064-200 - Uberaba (MG), Brasil E-mail: patricia_ambiental@hotmail.com

Recebido em: 19/05/16 - Aceito em: 16/12/16 - Reg. ABES: 163737 
Existem vários métodos de medição de sedimentos. A sedimentometria é a disciplina que trata das medições da descarga sólida, ou seja, da quantidade de sedimentos transportada pelos rios. As medições diretas em rios são realizadas de forma instantânea. A medição de descarga sólida pode ser feita por métodos diretos e indiretos; é dividida em medição de descarga sólida em suspensão, responsável, na maioria dos casos, por cerca de $90 \%$ da descarga total; e em medição de descarga sólida de arrasto, que fica com o restante do percentual (CARVALHO, 2008). Há casos em que esse percentual é bem diferente, mas na maioria das vezes esses valores se aplicam.

Para a mesma composição de sedimentos de fundo, partículas se arrastam, rolam ou se movem por saltação se a velocidade for baixa; à medida que a velocidade aumenta, parte desse sedimento é carregada para uma zona na qual o fluxo é maior, passando a ser sedimento em suspensão. O restante se mantém na camada mais profunda do corpo hídrico (CARVALHO, 2008; SUBCOMMITTEE ON SEDIMENTATION, 1965; WMO, 1981).

A descarga sólida em suspensão, que corresponde ao transporte de sedimentos em suspensão, é de difícil determinação, já que a distribuição do sedimento na seção do rio não é uniforme. Segundo Vanoni (1977), as forças que atuarão na partícula de sedimento variam de acordo com o tamanho da partícula (granulometria), a forma de escoamento (laminar ou turbulento), a velocidade de corrente, os obstáculos no leito, a temperatura da água, entre outros. Por esse motivo, a medição em um ponto não representa a concentração da seção. Desse modo, é necessária a realização de amostragens ao longo da seção, pontuais ou verticais, em um número adequado para a caracterização da seção (CARVALHO, 2008). Esse tipo de amostragem requer muito tempo em campo, e, dependendo do tamanho do rio ou do reservatório, a coleta de amostras pode durar dias, tornando alto o custo e até mesmo inviabilizando a amostragem.

Os métodos determinísticos levam em consideração a maior parte das variáveis que interferem no processo para quantificar os sedimentos em uma seção e, assim, verificar o transporte de sedimentos e assoreamento em reservatórios. Porém, além do alto custo de mensurar todas as variáveis, esses métodos produzem resultados com erros consideráveis. Dessa forma, é necessário o desenvolvimento de novas técnicas que reduzam o custo do monitoramento e garantam a qualidade da estimativa. Neste trabalho, é apresentada a utilização da teoria da entropia para determinar o perfil de concentração de sedimentos em reservatórios. Com apenas três pontos de monitoramento, é possível encontrar os parâmetros necessários para a estimativa, reduzindo consideravelmente o tempo e o custo de amostragens. Assim, é possível viabilizar campanhas de monitoramento de sedimentos e aumentar a rede de monitoramento com um custo menor.

\section{Teoria da entropia}

O físico francês Carnot, em 1824, vislumbrou a Segunda Lei da Termodinâmica em seus estudos sobre o fluxo de energia. O austríaco Ludwig Boltzmann, em 1877, introduziu, pela primeira vez, o conceito estatístico de entropia, estabelecendo uma relação direta entre a entropia e a desordem molecular de um processo térmico aleatório (RESNICK; HALLIDAY, 2008). Segundo Cápek e Sheehan (2004), a entropia é uma medida macroscópica quantitativa da desordem microscópica.

Em 1948, Shannon propôs uma teoria com bases matemáticas mais sólidas; ele estabeleceu uma conexão entre entropia e sequências típicas que permitiu a solução de inúmeros problemas nas áreas de codificação e transmissão de dados nos sistemas de comunicação em geral. De acordo com o autor citado, a entropia é uma medida da informação ou do grau de incerteza que se tem sobre um determinado sistema (SHANNON, 1948). O conceito de entropia já é bem estabelecido e usado na estatística e na teoria da informação, sendo quantificado em termos de probabilidade (Equação 1):

$H(X)=-\Sigma p\left(X_{j}\right) \operatorname{Lnp}\left(X_{j}\right)$

Em que:

$\mathrm{p}\left(\mathrm{X}_{\mathrm{f}}\right)$ é a probabilidade de o sistema estar no estado X com valores de $\left\{X_{j}, j=1,2, \ldots\right\}$.

De acordo com a teoria da informação, a informação contida nos dados é definida pela Equação 2:

$\operatorname{Ln} \frac{p(X j / \dot{X} j)}{p(X j)}$

A probabilidade de $\mathrm{X}$ varia de 0 a 1: quanto menor a probabilidade $\mathrm{p}\left(\mathrm{X}_{\mathrm{I}}\right)$ maior é o conteúdo de informação dos dados. Quando se sabe todo o conteúdo de informação, tem-se um caso determinístico. Todavia, a entropia $\mathrm{H}$ definida pela Equação 1 é o conteúdo médio de informação de uma amostra de dados. Se a variável X é contínua, a entropia pode ser expressa pela Equação 3:

$H(X)=-\int p(X) \ln p(X) d X$

O limite superior da distribuição de probabilidade uniforme de X mostra ser a máxima entropia. Na realidade, devido às várias restrições, a distribuição de probabilidade frequentemente não é uniforme. Ela é 0 nos casos puramente determinísticos em que a função conjunta de probabilidade $\mathrm{p}\left(\mathrm{X}_{\mathrm{i}}\right)=1$ e $\left(\mathrm{X}_{\mathrm{i}}\right)=0$, para todo i diferente de $\mathrm{j}$. A maximização 
da entropia do sistema fará com que a distribuição de probabilidade uniforme seja possível, desde que atenda às restrições.

O conceito de entropia, como usado na Teoria da Informação, fornece o grau de incerteza de um resultado específico em um processo, podendo, então, ser utilizado para o tratamento de variáveis hidrológicas (MUKHOPADHYAY; KHAN, 2015) e processos fluviais (GUOBIN; LINA, 2013). A concepção da probabilidade baseada na entropia tem sido utilizada na distribuição vertical de velocidades, concentração de sedimentos (CUI; SINGH, 2014; LUO; SINGH, 2011; SINGH; CUI, 2015), entre outras aplicações.

Uma grande limitação da aplicação da teoria da entropia é o número elevado de parâmetros a serem utilizados no modelo. Portanto, foi apresentada uma relação entre dois parâmetros, processo de redução das variáveis, para viabilizar seu uso e facilitar a estimativa da concentração de sedimentos em reservatórios.

\section{METODOLOGIA}

\section{Área de estudo}

Para a realização do presente estudo, foram utilizados os dados de concentração de sedimentos coletados por Santos (2015) a montante, bem como no reservatório da Central Hidrelétrica de Mogi-Guaçu (PCH. PH.SP.001479-6.01), localizado na Bacia Hidrográfica do Mogi-Guaçu (22022"45,6"' S; 46053"59,1"'” W).

O reservatório possui área de $5,73 \mathrm{~km}^{2}$ e volume de $32,89 \times 10^{6} \mathrm{~m}^{3}$. O entorno do reservatório é utilizado principalmente para o cultivo de cana-de-açúcar e há pequenas áreas de pastagem e plantio de café. A área de drenagem da bacia do reservatório apresenta alta suscetibilidade à erosão. Foram selecionados os pontos que possuíam, no mínimo, os dados da concentração na superfície e no fundo, e um valor intermediário para a modelagem.

Foram realizadas duas coletas identificadas após a numeração do ponto como um ou dois: o primeiro em janeiro, representando o período chuvoso, e o segundo em março, representando o período de estiagem. Os perfis amostrados estavam localizados na vertical de maior profundidade e seção retilínea. Isso é necessário, nessas condições, pelo fato de o fluxo de sedimentos ser unidimensional.

\section{Metodologia para a estimativa da concentração de sedimentos}

Para estimar a distribuição da concentração de sedimentos, foi utilizado o modelo probabilístico baseado na teoria da entropia de Cui e Singh (2014) (Equação 4), de acordo com a Figura 1:

$\mathrm{c}=\frac{\lambda_{*}}{\lambda_{1}}+\frac{1}{\lambda_{1}} \frac{\mathrm{m}}{\mathrm{m}-1}\left\{-\lambda_{1} \frac{\mathrm{m}-1}{\mathrm{~m}}\left(1-\frac{\mathrm{y}}{\mathrm{h}_{0}}\right)^{\mathrm{a}}+\left[\frac{\mathrm{m}-1}{\mathrm{~m}}\left(\lambda_{*}+\lambda_{1} \mathrm{c}_{\mathrm{h}}\right)\right]^{\frac{\mathrm{m}}{\mathrm{m}-1}}\right\}^{\frac{\mathrm{m}-1}{\mathrm{~m}}}$
Sendo $\lambda_{*}=\frac{1}{\mathrm{~m}-1}-\lambda_{1}$

Em que:

c é a concentração de sedimentos em uma distância vertical y, adimensional;

$\mathrm{c}_{\mathrm{m}}$ é o valor máximo de $\mathrm{c}$ ou concentração no leito, adimensional;

$\mathrm{c}_{\mathrm{h}}$ é a concentração na superfície da água, adimensional;

m é o parâmetro de entropia, adimensional;

$\lambda_{1}$ é o multiplicador de Lagrange, adimensional;

$\mathrm{h}_{0}$ é a profundidade do fluxo, em $\mathrm{m}$;

a é o parâmetro relacionado com as características das partículas de sedimento, adimensional.

Dessa forma, a Equação 4 pode ser reescrita para um ponto qualquer (Equação 5) e para o de maior concentração de sedimento, no ponto mais profundo (Equação 6).

$\mathrm{c}=\frac{\lambda_{*}}{\lambda_{1}}+\frac{1}{\lambda_{1}} \frac{\mathrm{m}}{\mathrm{m}-1}\left\{-\lambda_{1} \frac{\mathrm{m}-1}{\mathrm{~m}}\left(1-\frac{\mathrm{y}}{\mathrm{h}_{0}}\right)^{\mathrm{a}}+\left[\frac{\mathrm{m}-1}{\mathrm{~m}}\left(\lambda_{*}+\lambda_{1} \mathrm{c}_{\mathrm{h}}\right)\right]^{\frac{\mathrm{m}}{\mathrm{m}-1}}\right\}^{\frac{\mathrm{m}-1}{\mathrm{~m}}}$

$\mathrm{c}_{\mathrm{m}}=\frac{\lambda_{*}}{\lambda_{1}}+\frac{1}{\lambda_{1}} \frac{\mathrm{m}}{\mathrm{m}-1}\left\{-\lambda_{1} \frac{\mathrm{m}-1}{\mathrm{~m}}(1)^{\mathrm{a}}+\left[\frac{\mathrm{m}-1}{\mathrm{~m}}\left(\lambda_{*}+\lambda_{1} \mathrm{c}_{\mathrm{h}}\right)\right]^{\frac{\mathrm{m}}{\mathrm{m}-1}}\right\}^{\frac{\mathrm{m}-1}{\mathrm{~m}}}$

Reorganizando a Equação 4 sugerida por Cui e Singh (2014), tem-se:

$\frac{1}{c_{\mathrm{m}}}=\frac{1}{\mathrm{c}}\left(\frac{\lambda_{*}}{\lambda_{1} \mathrm{c}_{\mathrm{m}}}-\frac{1}{\lambda_{1} \mathrm{c}_{\mathrm{h}}} \frac{\mathrm{m}}{\mathrm{m}-1}\left\{-\lambda_{1} \frac{\mathrm{m}-1}{\mathrm{~m}}\left(1-\frac{\mathrm{y}}{\mathrm{h}}\right)^{\mathrm{a}}\left[+\frac{\mathrm{m}-1}{\mathrm{~m}}\left(\lambda_{*}-\lambda_{1} \mathrm{c}_{\mathrm{h}}\right)\right]^{\frac{\mathrm{m}}{\mathrm{m}-1}}\right\}^{\frac{\mathrm{m}-1}{\mathrm{~m}}}\right)$

Invertendo a Equação 6 deduzida neste trabalho, tem-se:

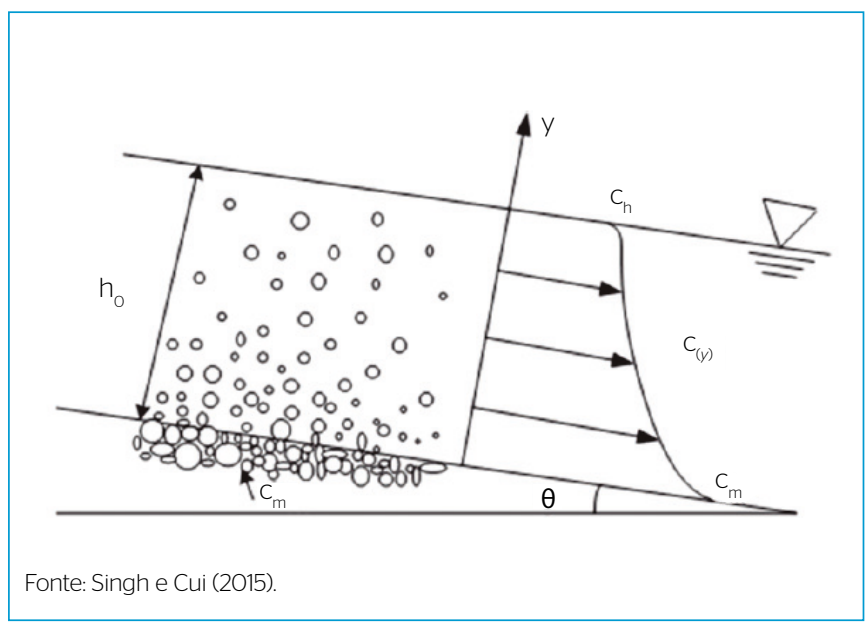

Figura 1 - Fluxo de sedimentos uniforme. 
$\frac{1}{\mathrm{c}_{\mathrm{m}}}=\frac{\lambda_{1}}{1+\lambda_{1}}\left\{-\lambda_{1} \frac{\mathrm{m}-1}{\mathrm{~m}}(1)^{\mathrm{a}}+\left[\frac{\mathrm{m}-1}{\mathrm{~m}}\left(\lambda_{*}-\lambda_{1} \mathrm{c}_{\mathrm{h}}\right)\right]^{\frac{\mathrm{m}}{\mathrm{m}-1}}\right\}^{\frac{1-\mathrm{m}}{\mathrm{m}}}$

Igualando as Equações 7 e 8, tem-se:

$$
\begin{aligned}
& \frac{1}{\mathrm{c}}\left(\frac{\lambda_{*}}{\lambda_{1} \mathrm{c}_{\mathrm{m}}}-\frac{1}{\lambda_{1} \mathrm{c}_{\mathrm{m}}} \frac{\mathrm{m}-1}{\mathrm{~m}}\left\{-\lambda_{1} \frac{\mathrm{m}-1}{\mathrm{~m}}\left(1-\frac{\mathrm{y}}{\mathrm{h}}\right)^{\mathrm{a}}+\left[\frac{\mathrm{m}-1}{\mathrm{~m}}\left(\lambda_{*}-\lambda_{1} \mathrm{c}_{\mathrm{h}}\right)\right]^{\frac{\mathrm{m}-1}{\mathrm{~m}-1}}\right\}^{\frac{\mathrm{m}-1}{\mathrm{~m}}}\right)= \\
& =\frac{\lambda_{1}(\mathrm{~m}-1)}{\lambda_{1}(\mathrm{~m}-1)-\mathrm{m}}\left\{-\lambda_{1} \frac{\mathrm{m}-1}{\mathrm{~m}}(1)^{\mathrm{a}}+\left[\frac{\mathrm{m}-1}{\mathrm{~m}}\left(\lambda_{*}-\lambda_{1} \mathrm{c}_{\mathrm{h}}\right)\right]^{\frac{\mathrm{m}}{\mathrm{m}-1}}\right\}^{\frac{1-\mathrm{m}}{\mathrm{m}}}
\end{aligned}
$$

Simplificando a igualdade expressa na Equação 9, considerando

$\mathrm{T} 1=-\lambda_{1} \frac{\mathrm{m}-1}{\mathrm{~m}}\left(1-\frac{\mathrm{y}}{\mathrm{D}}\right)^{\mathrm{a}}+\left[\frac{\mathrm{m}-1}{\mathrm{~m}}\left(\lambda_{*}-\lambda_{1} \mathrm{c}_{\mathrm{h}}\right)\right]^{\frac{\mathrm{m}}{\mathrm{m}-1}} \mathrm{e}$

$\mathrm{T} 2=-\lambda_{1} \frac{\mathrm{m}-1}{\mathrm{~m}}(1)^{\mathrm{a}}+\left[\frac{\mathrm{m}-1}{\mathrm{~m}}\left(\lambda_{*}-\lambda_{1} \mathrm{c}_{\mathrm{h}}\right)\right]^{\frac{\mathrm{m}}{\mathrm{m}-1}}$, tem-se a Equação $10:$

$\operatorname{In}\left(\frac{\left(1+\lambda_{1}\right)^{2}}{\lambda_{1}(1+\mathrm{CCm})}\right)+\frac{\mathrm{m}-1}{\mathrm{~m}} \operatorname{In}(\mathrm{T} 1 \mathrm{~T} 2)=0$

Dessa forma, foi obtido um sistema com três incógnitas $-\lambda_{1}$, " $a$ " e " $m$ " - e duas equações. As variáveis $c_{h}$, c e c $c_{m}$, bem como y e $h_{0}$ devem ser obtidas nos dados de campo. Foi encontrada a relação entre os parâmetros $m=\exp F(c)$. Essa equação relaciona "m" $\operatorname{com} \lambda_{1}$, tornando possível a solução numérica da Equação 10. Então, para cada profundidade, obteve-se um valor de concentração, sendo necessário encontrar apenas os valores de $\lambda_{1}$ e de "a".

A Equação 10 é a base do método desenvolvido. Diferente dos trabalhos anteriores, a concentração mínima não é considerada 0 , e sim a concentração superficial.

\section{Avaliação da eficácia do modelo}

A eficácia do modelo foi avaliada aplicando os seguintes coeficientes estatísticos: coeficiente de Nash-Sutcliffe (NSE); coeficiente de determinação $\left(\mathrm{R}^{2}\right)$; desvio entre vazões observadas e simuladas (D), PBIAS (percentual de viés dos dados simulados com os dados observados), relação entre a raiz quadrada média do erro e o desvio padrão dos dados medidos (RSR) e raiz do erro médio quadrático (RMSE).

De acordo com Molnar (2011), o valor de NSE indica o ajustamento dos dados simulados aos observados na reta $1: 1$, podendo variar de $-\infty$ a 1. Molnar (2011) apresentou a seguinte classificação para esse coeficiente, utilizando passo diário de simulação: NSE >0,8, o modelo é considerado excelente; $0,8<\mathrm{NSE}<0,6$, muito bom; $0,4<\mathrm{NSE}<0,6$, bom; entre 0,4 e 0,2, satisfatório; e $<0,2$, insuficiente. Segundo Moriasi et al. (2007), valores de NSE acima de 0,5 qualificam o modelo para simulação. Segundo esses autores, valores de $\mathrm{R}^{2}$ acima de 0,5 são considerados aceitáveis.

O valor de D significa a tendência média das estimativas produzidas pelo modelo e, quando positivo, expressa tendência de superestimativa; quando negativo, de subestimativa. Viola et al. (2012) apresentam os seguintes intervalos e respectivas interpretações de D: <10\%, muito bom; entre 10 e 15\%, bom; entre 15 e 25\%, satisfatório; e $>25 \%$, o modelo produz estimativas inadequadas no tocante à tendência.

PBIAS também mede o desvio dos dados. Quando positivo, o modelo tende a superestimar os dados, e, quando negativo, a subestimar os dados simulados em relação aos medidos. Um modelo ideal teria o valor 0 . Em geral, a simulação do modelo pode ser julgada como satisfatória se NSE $>0,50$ e $\mathrm{RSR}<0,70$, e se $\mathrm{PBIAS} \leq 55 \%$ para os sedimentos.

\section{RESULTADOS E DISCUSSÃO}

As variações das concentrações de sedimentos estimadas e observadas com diferentes valores de a e com a equação de Cui e Singh (2014) com $m=3$ mostram que a variação da concentração de sedimentos ao longo do perfil depende do valor de a (Figura 2).

Na Figura 2, os pontos 6, 12 e 20 foram escolhidos aleatoriamente para mostrar a variação do parâmetro "a”. Foram simulados vários valores de a e adotados os que apresentaram o melhor $\mathrm{R}^{2}$.

Comparando os resultados com o modelo adotado por Cui e Singh (2014) e utilizando o parâmetro de entropia $\mathrm{m}=3$, conforme sugerido pelos autores, observa-se que o modelo utilizado neste trabalho é superior, pois representa melhor o perfil de concentração de sedimentos (Figuras 2 e 3). Os valores encontrados acompanham as alterações de concentração ao longo do perfil, diferente do modelo de Cui e Singh (2014), que, mesmo com alteração do parâmetro "a", apresenta apenas o valor próximo ao médio do perfil. De acordo com Cui e Singh (2014), o expoente "a" tem relação direta com o diâmetro das partículas em suspensão.

A partir das simulações realizadas variando o valor de "a" e de $\lambda_{1}$, foram adotados os resultados que apresentaram os maiores valores de NSE e $\mathrm{R}^{2}$, respectivamente (Tabela 1). Os perfis de concentração analisados possuem variações na concentração de sedimentos (de 10 a $140 \mathrm{~g} \mathrm{~L}^{-1}$ ) e na profundidade (de 1 a $10 \mathrm{~m}$ ), dependendo da localização a montante e dentro do reservatório (Figura 3).

Com três pontos de amostragem, concentrações mínima e máxima e um ponto intermediário, é possível traçar o perfil de concentração de sedimentos, independentemente de outras variáveis. 
Foi verificado que é possível modelar o perfil de concentração de sedimentos em reservatório com diferentes situações de fluxo de maneira satisfatória (Tabela 1). Os pontos P1_2, P8_2, P12_2, P14_2 e P19_2 representaram o período de estiagem (março), e os pontos P6_1, P13_1 e P20_1, o período de cheias.

Apenas os pontos P6_2, P16_2 e P19_2 não tiveram resultados satisfatórios, pois o material de fundo foi suspenso devido às dragagens que eram realizadas próximas ao local de coleta das amostras. O ponto P16_1 foi descartado, visto que apresentou os valores mínimos de concentração de sedimentos no fundo e máximos na superfície. Fica claro que a interferência da dragagem trouxe prejuízo aos resultados desses perfis. De qualquer maneira, é interesse mostrar esses resultados para identificar que a modelagem não consegue prever o perfil de concentração de sedimentos em áreas com revolvimento de fundo por qualquer razão. Apesar de a determinação da concentração de sedimentos do ponto P6_2 não

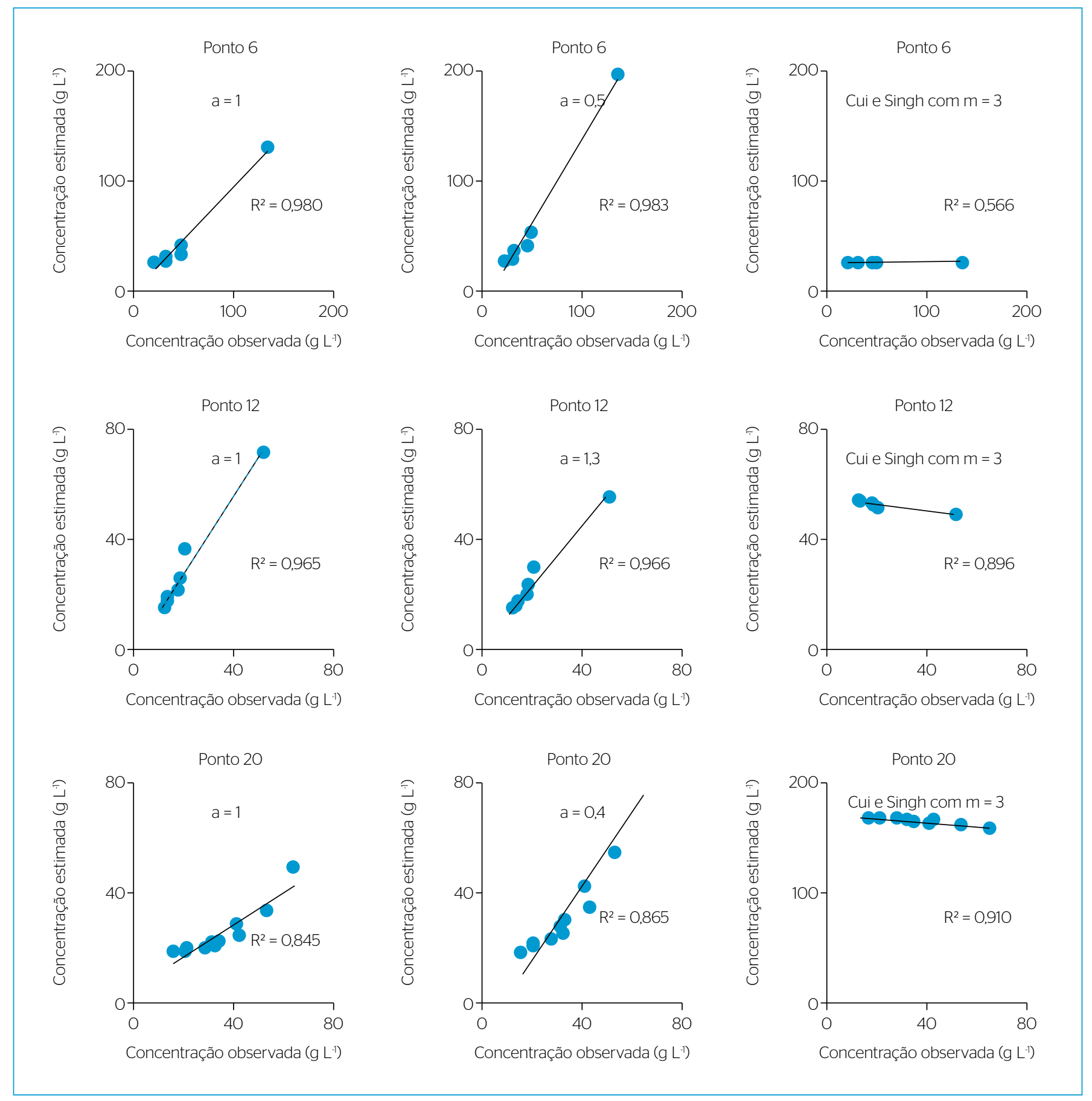

Figura 2 - Concentração de sedimentos estimada e observada nos pPontos 6, 12 e 20 com diferentes valores de "a" e com a equação de Cui e Singh (2014) com m=3. 

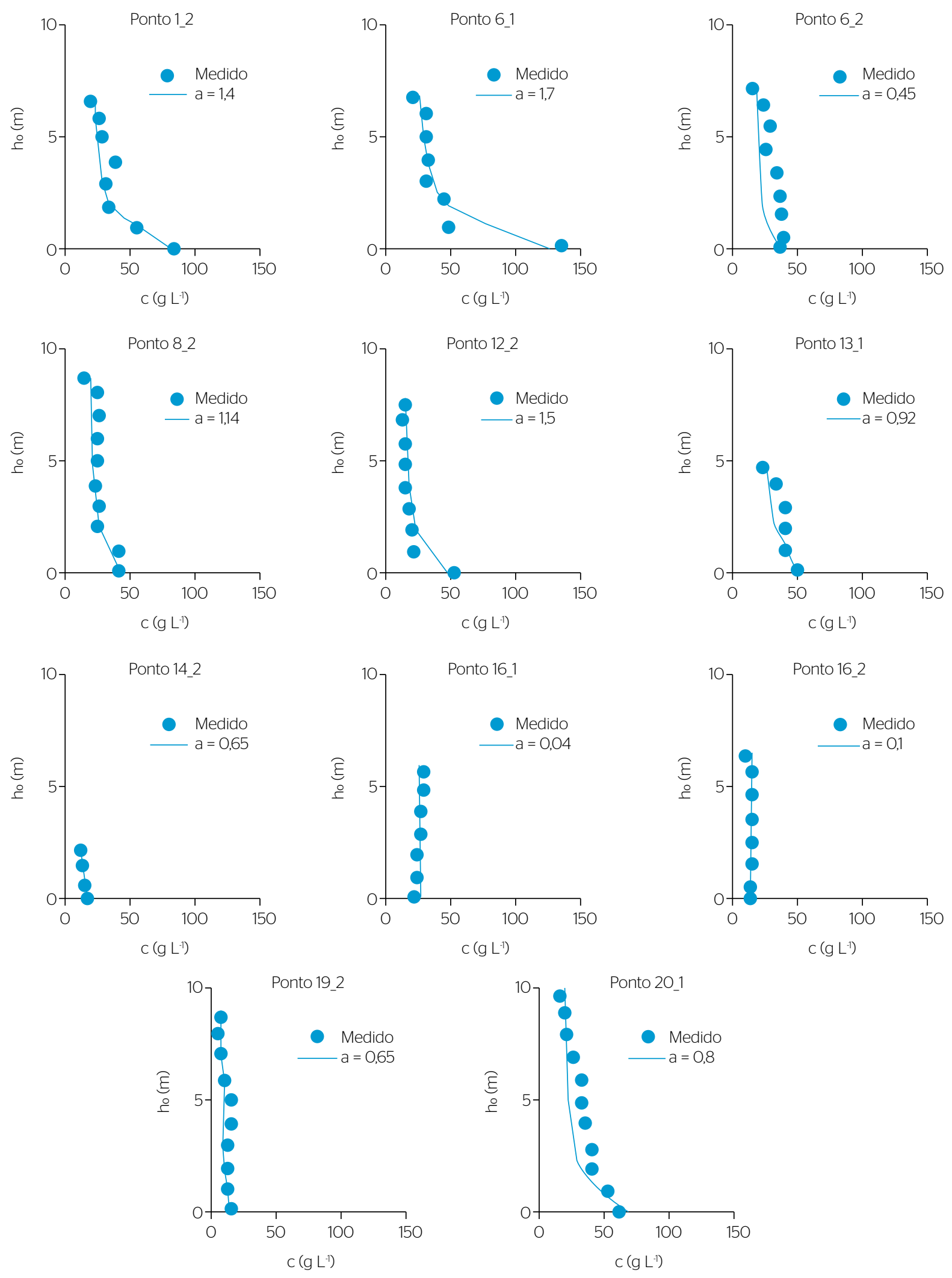

Figura 3 - Variação da concentração de sedimentos e profundidade dos perfis. 
Tabela 1 - Resumo dos resultados das simulações.

\begin{tabular}{|c|c|c|c|c|c|c|c|c|c|c|}
\hline Perfil & D (\%) & $D^{* *}$ & NSE & NSE*** & $\mathrm{R}^{2}$ & $\mathrm{R}^{2 *}$ & PBIAS (\%) & PBIAS* & RSR & $\mathrm{RSR}^{*}$ \\
\hline P6_1 & -18 & Satisfatório & $-0,49$ & I & 0,39 & I & 21 & S & 1,16 & I \\
\hline P8_2 & -4 & Muito bom & 0,76 & Muito bom & 0,82 & S & 5 & $S$ & 0,46 & S \\
\hline P12_2 & 22 & Satisfatório & 0,67 & Muito bom & 0,80 & S & -17 & S & 0,62 & $S$ \\
\hline P16_2 & -1 & Muito bom & $-0,04$ & 1 & 0,00 & I & 2 & S & 1,48 & I \\
\hline P19_2 & -10 & Satisfatório & 0,28 & I & 0,30 & I & 15 & S & 1,15 & I \\
\hline P6_1 & 9 & Muito bom & 0,85 & Excelente & 0,87 & S & -6 & S & 0,33 & S \\
\hline P13_1 & -7 & Muito bom & 0,49 & Bom & 0,50 & S & 9 & S & 0,69 & S \\
\hline
\end{tabular}

*Classificação de acordo com Moriasi et al. (2007); **Classificação de acordo com Liew et al. (2003 apud VIOLA et al., 2012); ***Classificação de acordo com Molnar (2011); D: desvio dos valores simulados dos observados; NSE: coeficiente de Nash-Sutcliffe; $\mathrm{R}^{2}$ : coeficiente de determinação; PBIAS: percentual de viés dos dados simulados com os dados observados; RSR: relação entre a raiz quadrada média do erro com o desvio padrão dos dados medidos; S: satisfatório; l: insatisfatório.

ser satisfatória devido ao revolvimento de fundo pelas dragagens, pode-se verificar que foi possível a sua determinação em outro momento (P6_1), já que esse ponto é o mesmo, e apenas a condição de coleta foi diferente.

O valor de D não ultrapassou $25 \%$ em nenhum caso, e isso significa a tendência média das estimativas produzidas pelo modelo. $O$ valor de PBIAS também não ultrapassou 55\%. Isso mostra que o modelo não superestimou nem subestimou os valores estimados.

A utilização da relação $m=\operatorname{expF}(c)^{\wedge} \lambda_{1}$, além de produzir melhores resultados, com $\mathrm{R}^{2}$ superior aos valores de Cui e Singh (2014) com $\mathrm{m}=3$, reduz o número de incógnitas a serem mensuradas, tornando possível a solução numérica e, consequentemente, diminuindo o tempo computacional.

\section{CONCLUSÕES}

A teoria da entropia, considerada eficiente pela simplicidade do método e pela redução do custo de amostragens, pode ser utilizada para simular o perfil de concentração de sedimentos em reservatórios. A utilização da relação entre os parâmetros facilita os cálculos, reduz o número de parâmetros modelados e, consequentemente, diminui o tempo computacional, além de representar melhor as variações da concentração de sedimentos ao longo do perfil.

\section{REFERÊNCIAS}

CAPEK, V.; SHEEHAN, D.P. (2004) Challenges to the second law of thermodynamics: Theory and experiment. Estados Unidos: Springer. $367 \mathrm{p}$.

CARVALHO, N.O. (2008) Hidrossedimentologia prática. 2. ed. Rio de Janeiro: Interciência. 599 p.

CUI, H.; SINGH, V.P. (2014) Suspended sediment concentration in open channels using tsallis entropy. Journal of Hydrologic Engineering, v. 19, p. 966-977.

GUOBIN, X.; LINA, Z. (2013) Analysis of fluvial process based on information entropy. Journal of Tianjin University Science and Technology, v. 43, p. 347-353.
LUO, H.; SINGH, V.P. (2011) Entropy theory for two-dimensional velocity distribution. Journal of Hydrologic Engineering, p. 303-315.

MOLNAR, P. (2011) "Calibration". Watershed Modelling, SS. Switzerland: Institute of Environmental Engineering, Chair of Hydrology and Water Resources Management, ETH Zürich.

MORIASI, D.N.; ARNOLD, J.G.; VAN LIEW, M.W.; BINGNER, R.L.; HARMEL, R.D.; VEITH, T.L. (2007) Model evaluation guidelines for systematic quantification of accuracy in watershed simulations. Transactions of the ASABE, v. 50, n. 3, p. 885-900.

MUKHOPADHYAY, B.; KHAN, A. (2015) Boltzmann-Shannon entropy and river flow stability within Upper Indus Basin in a changing climate. International Journal of River Basin Management, v. 13, p. 87-95. 
RESNICK; HALLIDAY. (2008) Fundamentos de Física: mecânica. 8. ed. Rio de Janeiro: LTC. v. 1.

SANTOS, B.B. (2015) Estudo da concentração de sedimentos em suspensão no reservatório de Mogi-Guaçu (SP). Dissertação (Mestrado) - Programa de Pós-Graduação em Ciências da Engenharia Ambiental, Escola de Engenharia de São Carlos, Universidade de São Paulo, São Carlos.

SHANNON, C.E. (1948) A Mathematical Theory of Communication. The Bell System Technical Journal, v. 27, p. 623-656.

SINGH, V.P.; CUI, H. (2015) Modeling sediment concentration in debris flow by Tsallis entropy. Physica A: Statistical Mechanics and its Applications, p. 49-58.
SUBCOMMITTEE ON SEDIMENTATION. (1965) Determination on fluvial sediment discharge. Report, n. 14. Minneapolis: Inter-Agency on Water Resources.

VIOLA, M.R.C.R.; MELLO, C.R.; GIONGO, M.; BESKOW, S.; SANTOS, A.F. (2012) Hydrological Modeling in a watershed of the Lower Araguaia River Basin, TO. Journal of Technology and Biodiversity, v. 3, n. 3, p. 38-47.

VANONI, V.A. (1977) Sedimentation engineering. New York: ASCE.

WORLD METEOROLOGICAL ORGANIZATION (WMO). (1981) Guide to hydrological practices. Geneva: WMO. v. 168. 